\title{
221. Note on the Asymptotic Normality of a Stochastic Process with Independent Increments
}

\author{
By Motoo HorI and Tetsuo FujImagari \\ Tokyo Institute of Technology
}

(Comm. by Kinjirô KUnUGi, M. J. A., Oct. 12, 1971)

1. In the present paper we are concerned with the continuous analogue of the classical central limit theorem. The Lindeberg theorem establishes necessary and sufficient conditions under which sums of mutually independent random variables are asymptotically normally distributed. We shall show that the normal convergence law of the Lindeberg type holds for a stochastic process with independent increments, which is essentially the continuous parameter version of a sequence of consecutive sums of mutually independent random variables. In some practical applications, it is of real importance to determine limiting distributions for continuous parameter processes with independent increments [1] [2].

2. Let $\left\{x_{t}, t \geq 0\right\}$ be a continuous parameter process with independent increments which is not necessarily temporally homogeneous. In what follows, we assume that $x_{0} \equiv 0$ and that there are no fixed points of discontinuity. As is well known [3], the characteristic function of $x_{t}$ has the form

$$
\left\{\begin{array}{l}
E\left(e^{i \zeta x t}\right)=e^{\varphi(\zeta, t)}, \\
\varphi(\zeta, t)=i \zeta m(t)-\frac{\zeta^{2}}{2} v^{2}(t)+\int_{-\infty}^{\infty}\left(e^{i \zeta u}-1-\frac{i \zeta u}{1+u^{2}}\right) \nu_{t}(d u) .
\end{array}\right.
$$

Here $m(t)$ is a continuous function of $t, v^{2}(t)$ is a non-negative, monotone non-decreasing, and continuous function of $t$, and $\nu_{t}(d u)$ $\equiv \int_{\tau=0}^{t} \nu(d \tau d u)$ is a measure on $(-\infty, \infty) \backslash\{0\}$ satisfying $\nu(\{t\} \times d u) \equiv 0$ and

$$
\int_{-\infty}^{\infty} \frac{u^{2}}{1+u^{2}} \nu_{t}(d u)<\infty
$$

The following lemma can be verified directly from the formula (1).

Lemma 1. If $E\left(x_{t}{ }^{2}\right)$ is finite, or equivalently,

$$
\int_{-\infty}^{\infty} u^{2} \nu_{t}(d u)<\infty
$$

for all $t>0$, then the expectation $\mu(t)=E\left(x_{t}\right)$ and the variance $\sigma^{2}(t)$ $=\operatorname{Var}\left(x_{t}\right)$ are given by

(4) $\mu(t)=m(t)+\int_{-\infty}^{\infty} \frac{u^{3}}{1+u^{2}} \nu_{t}(d u), \quad \sigma^{2}(t)=v^{2}(t)+\int_{-\infty}^{\infty} u^{2} \nu_{t}(d u)$. 
We now obtain a continuous analogue to the classical central limit theorem.

Theorem 1. Let $\sigma^{2}(t)<\infty$ for all $t>0$. In order that for any real $x$

$$
P\left\{\frac{x_{t}-\mu(t)}{\sigma(t)} \leq x\right\} \rightarrow \Phi(x) \equiv \frac{1}{\sqrt{2 \pi}} \int_{-\infty}^{x} e^{-z^{2 / 2}} d z
$$

as $t \rightarrow \infty$, it is necessary and sufficient that the Lindeberg type condition

$$
\frac{1}{\sigma^{2}(t)} \int_{|u|>\delta \sigma(t)} u^{2} \nu_{t}(d u) \rightarrow 0, \quad t \rightarrow \infty
$$

be satisfied for every fixed $\varepsilon>0$.

Proof. Defining $\psi(\zeta, t)$ by

$$
E\left[e^{i \zeta\left(x_{t}-\mu(t)\right) / \sigma(t)}\right]=e^{\psi(\zeta, t)},
$$

we have from (1) and (4)

$$
\begin{aligned}
\psi(\zeta, t)= & -i \zeta \frac{\mu(t)}{\sigma(t)}+\varphi\left[\frac{\zeta}{\sigma(t)}, t\right] \\
= & -i \zeta \frac{\mu(t)}{\sigma(t)}+i \zeta \frac{m(t)}{\sigma(t)}-\frac{\zeta^{2}}{2} \frac{v^{2}(t)}{\sigma^{2}(t)} \\
& +\int_{-\infty}^{\infty}\left\{e^{i \zeta u / \sigma(t)}-1-\frac{i \zeta u / \sigma(t)}{1+u^{2}}\right\} \nu_{t}(d u) \\
= & -\frac{\zeta^{2}}{2}+\int_{-\infty}^{\infty} h(\zeta, u, t) \nu_{t}(d u),
\end{aligned}
$$

where

$$
h(\zeta, u, t)=e^{i \zeta u / \sigma(t)}-1-\frac{i \zeta u}{\sigma(t)}+\frac{\zeta^{2} u^{2}}{2 \sigma^{2}(t)} .
$$

The assertion (5) is thus equivalent to the statement that for all $\zeta$

$$
\lim _{t \rightarrow \infty} \int_{-\infty}^{\infty} h(\zeta, u, t) \nu_{t}(d u)=0 .
$$

Sufficiency. It is easily shown that

$$
\begin{aligned}
& |h(\zeta, u, t)|=\left|e^{i \zeta u / \sigma(t)}-1-\frac{i \zeta u}{\sigma(t)}+\frac{\zeta^{2} u^{2}}{2 \sigma^{2}(t)}\right| \leq \frac{\left|\zeta^{3} u^{3}\right|}{6 \sigma^{3}(t)} \\
& |h(\zeta, u, t)| \leq\left|e^{i \zeta u / \sigma(t)}-1-\frac{i \zeta u}{\sigma(t)}\right|+\frac{\zeta^{2} u^{2}}{2 \sigma^{2}(t)} \leq \frac{\zeta^{2} u^{2}}{\sigma^{2}(t)}
\end{aligned}
$$

Therefore,

$$
\begin{aligned}
& \left|\int_{-\infty}^{\infty} h(\zeta, u, t) \nu_{t}(d u)\right| \\
& \quad \leq \frac{|\zeta|^{3}}{6 \sigma^{3}(t)} \int_{|u| \leqslant \varepsilon \sigma(t)}|u|^{3} \nu_{t}(d u)+\frac{\zeta^{2}}{\sigma^{2}(t)} \int_{|u|>\delta \sigma(t)} u^{2} \nu_{t}(d u) \\
& \quad \leq \frac{\varepsilon|\zeta|^{3}}{6}+\frac{\zeta^{2}}{\sigma^{2}(t)} \int_{|u|>\varepsilon \sigma(t)} u^{2} \nu_{t}(d u)
\end{aligned}
$$

The factor of $\zeta^{2}$ is the expression occurring in the condition (6), and $\varepsilon$ can be chosen arbitrarily small, so that the integral on the left side tends to 0 . 
Necessity. Taking the real part of (10), we get

$$
\begin{gathered}
\frac{\zeta^{2}}{2 \sigma^{2}(t)} \int_{-\infty}^{\infty} u^{2} \nu_{t}(d u)-\int_{|u| \leq \varepsilon \sigma(t)}\left\{1-\cos \frac{\zeta u}{\sigma(t)}\right\} \nu_{t}(d u) \\
=\int_{|u|>\varepsilon \sigma(t)}\left\{1-\cos \frac{\zeta u}{\sigma(t)}\right\} \nu_{t}(d u)+o(1)
\end{gathered}
$$

as $t \rightarrow \infty$. By the inequalities

$$
1-\cos \frac{\zeta u}{\sigma(t)} \leq \frac{\zeta^{2} u^{2}}{2 \sigma^{2}(t)}, \quad 1-\cos \frac{\zeta u}{\sigma(t)} \leq 2,
$$

it follows that

$$
\begin{aligned}
& \frac{\zeta^{2}}{2 \sigma^{2}(t)} \int_{-\infty}^{\infty} u^{2} \nu_{t}(d u)-\frac{\zeta^{2}}{2 \sigma^{2}(t)} \int_{|u| \leq \varepsilon \sigma(t)} u^{2} \nu_{t}(d u) \\
& \quad \leq 2 \int_{|u|>\sigma \sigma(t)} \nu_{t}(d u)+o(1) \leq \frac{2}{\varepsilon^{2}}+o(1) .
\end{aligned}
$$

Dividing (15) by $\zeta^{2} / 2$ we see that

$$
\frac{1}{\sigma^{2}(t)} \int_{|u|>\delta \sigma(t)} u^{2} \nu_{t}(d u) \leq \frac{4}{\varepsilon^{2} \zeta^{2}}+o(1)
$$

The left side is independent of $\zeta$ while the right side can be made arbitrarily small by choosing $\zeta$ sufficiently large. Hence the left side tends to 0 , which completes the proof.

Remark. Theorem 1 is simpler than the Lindeberg theorem for sums of independent random variables, since in the continuous parameter case the condition that normalized summands are individually negligible or infinitesimal [4] is automatically satisfied. holds.

Corollary 1. If the process $\left\{x_{t}\right\}$ is homogeneous in time, then (5)

This is an immediate consequence of Theorem 1 with $\sigma^{2}(t)=t \sigma^{2}(1)$ and $\nu_{t}(d u)=t \nu_{1}(d u)$.

Corollary 2. Suppose that for some $k>2$

$$
\int_{-\infty}^{\infty}|u|^{k} \nu_{t}(d u)<\infty
$$

Then, the condition (6) is satisfied whenever

$$
\frac{1}{\sigma^{k}(t)} \int_{-\infty}^{\infty}|u|^{k} \nu_{t}(d u) \rightarrow 0
$$

as $t \rightarrow \infty$. (Ljapunov's sufficient condition).

The proof proceeds as follows:

$$
\begin{aligned}
\frac{1}{\sigma^{2}(t)} \int_{|u|>\varepsilon \sigma(t)} u^{2} \nu_{t}(d u) & \leq \frac{1}{\sigma^{2}(t)} \int_{|u|>\varepsilon \sigma(t)} u^{2}\left|\frac{u}{\varepsilon \sigma(t)}\right|^{k-2} \nu_{t}(d u) \\
& \leq \frac{1}{\varepsilon^{k-2} \sigma^{k}(t)} \int_{-\infty}^{\infty}|u|^{k} \nu_{t}(d u) \rightarrow 0 .
\end{aligned}
$$

Theorem 2. If (6) holds for each fixed $\varepsilon>0$ and $\nu_{t}\{(-\infty, \infty)\} \neq 0$, then $\sigma(t) \rightarrow \infty$ as $t \rightarrow \infty$.

Proof. Suppose that there exists a number $a$ such that $\sigma(t) \leq a<\infty$ 
for all $t$. From (6) we find

$$
\int_{|u|>\delta a} u^{2} \nu_{t}(d u) \leq \int_{|u|>\delta \sigma(t)} u^{2} \nu_{t}(d u) \rightarrow 0, \quad t \rightarrow \infty .
$$

Since $\varepsilon>0$ is arbitrary and $\nu_{t}(d u)$ is non-decreasing in $t$, it can be concluded that

$$
\int_{-\infty}^{\infty} u^{2} \nu_{t}(d u) \equiv 0
$$

which leads to $\nu_{t}\{(-\infty, \infty)\} \equiv 0$. This is a contradiction.

3. We next consider a continuous parameter process $\left\{X_{t}, t \geq 0\right\}$ with independent increments which has no fixed points of discontinuity. Note that $X_{0}$ is not necessarily required to be zero. However, the characteristic function of the random variable $x_{t}=X_{t}-X_{0}$ may be written in the same form as (1).

Lemma 2. Let $E\left(x_{t}^{2}\right)$ and $E\left(X_{t}^{2}\right)$ be finite, and put $\sigma^{2}(t)=\operatorname{Var}\left(x_{t}\right)$ and $S^{2}(t)=\operatorname{Var}\left(X_{t}\right)$. Then $S(t) \rightarrow \infty$ as $t \rightarrow \infty$, if and only if $\sigma(t) \rightarrow \infty$. In this case, $S(t) \sim \sigma(t)$.

Proof. Let us consider the covariances $\operatorname{Cov}\left(X_{0}, x_{t}\right)$ and $\operatorname{Cov}\left(X_{0}\right.$, $\left.X_{t}\right)$. It is evident that

$$
\begin{aligned}
& \left|S^{2}(0)-S^{2}(t)+\sigma^{2}(t)\right|=2\left|\operatorname{Cov}\left(X_{0}, x_{t}\right)\right| \leq 2 S(0) \sigma(t), \\
& \left|S^{2}(0)+S^{2}(t)-\sigma^{2}(t)\right|=2\left|\operatorname{Cov}\left(X_{0}, X_{t}\right)\right| \leq 2 S(0) S(t),
\end{aligned}
$$

whence either $\sigma(t) \rightarrow \infty$ or $S(t) \rightarrow \infty$ implies $\sigma(t) / S(t) \rightarrow 1$.

Hereafter we shall add the following assumption : $X_{0}$ is independent of $x_{t}=X_{t}-X_{0}$ for all $t>0$; namely, $\left\{X_{t}\right\}$ forms a spatially homogeneous Markov process.

Theorem 3. Suppose that $S(t)<\infty$ and $S(t) \rightarrow \infty$ as $t \rightarrow \infty$, and put $M(t)=E\left(X_{t}\right)$. Then for every fixed $x$,

$$
P\left\{\frac{X_{t}-M(t)}{S(t)} \leq x\right\} \rightarrow \Phi(x), \quad t \rightarrow \infty,
$$

if and only if for any given $\varepsilon>0$

$$
\frac{1}{S^{2}(t)} \int_{|u|>S S(t)} u^{2} \nu_{t}(\dot{d} u) \rightarrow 0, \quad t \rightarrow \infty .
$$

Proof. We start from the obvious equality

$$
\frac{X_{t}-M(t)}{S(t)}=\frac{x_{t}-\mu(t)}{\sigma(t)} \frac{\sigma(t)}{S(t)}+\frac{X_{0}-M(0)}{S(t)} .
$$

Lemma 2 means that the factor $\sigma(t) / S(t)$ in (25) tends to 1 as $t \rightarrow \infty$. From the independence of $x_{t}$ and $X_{0}$, therefore, it follows that the assertion (23) is equivalent to (5). On the other hand, the two conditions (6) and (24) are equivalent to each other, because for large $t$ and any $\varepsilon>0$

$$
\left\{\begin{array}{l}
\frac{1}{S^{2}(t)} \int_{|u|>\delta S(t)} u^{2} \nu_{t}(d u) \leq \frac{\sigma^{2}(t)}{S^{2}(t)} \frac{1}{\sigma^{2}(t)} \int_{|u|>\varepsilon \sigma(t)} u^{2} \nu_{t}(d u), \\
\frac{1}{\sigma^{2}(t)} \int_{|u|>\delta \sigma(t)} u^{2} \nu_{t}(d u) \leq \frac{S^{2}(t)}{\sigma^{2}(t)} \frac{1}{S^{2}(t)} \int_{|u|>(\varepsilon / 2) S(t)} u^{2} \nu_{t}(d u) .
\end{array}\right.
$$


The validity of this theorem is thus guaranteed by Theorem 1 .

Theorem 4. (a) In the case where $\nu_{t}\{(-\infty, \infty)\} \neq 0$, the condition (24) implies that $S(t) \rightarrow \infty$ as $t \rightarrow \infty$.

(b) Suppose that (23) holds and $\lim _{t \rightarrow \infty} S(t)<\infty$. Then $\nu_{t}\{(-\infty, \infty)\}$ $\equiv 0$; in other words, $X_{t}$ is continuous in $t$ with probability one. Furthermore, $X_{0}$ has a normal distribution.*)

(c) If $\nu_{t}\{(-\infty, \infty)\} \equiv 0$ and $X_{0}$ is normally distributed, then the statement (23) is true.

Proof. The proof of $(a)$ is exactly similar to that of Theorem 2. The assertion $(c)$ requires no comment. To prove $(b)$ we consider the characteristic function

(27) $\quad E\left[e^{i \zeta S(t) / \sigma(t) \cdot\left(X_{t}-M(t)\right) / S(t)}\right]=E\left[e^{i \zeta\left(x_{t}-\mu(t)\right) / \sigma(t)}\right] \cdot E\left[e^{i \zeta\left(X_{0}-M(0)\right) / \sigma(t)}\right]$.

The left side converges to $\exp \left[-(B / A)^{2} \cdot \zeta^{2} / 2\right]$, where $A=\lim _{t \rightarrow \infty} \sigma(t)<\infty$ and $B=\lim _{t \rightarrow \infty} S(t)<\infty$. In addition, the second factor on the right converges to $E\left[\exp \left(i \zeta\left\{X_{0}-M(0)\right\} / A\right)\right]$. Hence the random variable $\left\{x_{t}-\mu(t)\right\} / \sigma(t)$ converges in law as $t \rightarrow \infty$. According to Cramér-Lévy's theorem [5], the assertion (5) holds and $X_{0}$ has a normal distribution, so that $\nu_{t}\{(-\infty, \infty)\} \equiv 0$ by Theorem 1 and Theorem 2 . The proof is accomplished.

Acknowledgement. The authors are much indebted to Prof. K. Sato for suggesting the proof of Theorem 2.

\section{References}

[1] Hori, M., and M. Uchida: Application of the theory of Markov processes to comminution. I. The case of discrete time parameter. Kōdai Math. Sem. Rep., 19, 174-188 (1967). Correction. ibid., 20, 125-126 (1968).

[2] Hori, M.: Application of the theory of Markov processes to comminution. II. The case of continuous time parameter (to be published).

[ 3 ] Ito, K.: Lectures on Stochastic Processes. Tata Institute of Fundamental Research, Bombay (1964).

[ 4 ] Gnedenko, B. V., and A. N. Kolmogorov: Limit Distributions for Sums of Independent Random Variables. Addison-Wesley, Reading (1954).

[5] Loève, M.: Probability Theory (3rd. ed.). Van Nostrand, Princeton (1963).

*) Here the term normal distribution includes the case of degenerate distributions. 